\title{
A comparison of the use of vacuum metal deposition versus cyanoacrylate fuming for visualisation of fingermarks and grab impressions on fabrics.
}

\author{
Joanna Fraser, Paul Deacon, Stephen Bleay, David H Bremner
}

\begin{abstract}
Both vacuum metal deposition (VMD) and cyanoacrylate fuming (CAF) are techniques used to visualise latent fingermarks on smooth non-porous surfaces such as plastic and glass. VMD was initially investigated in the 1970 s as to its effectiveness for visualising prints on fabrics, but was abandoned when radioactive sulphur dioxide was found to be more effective. However, interest in VMD was resurrected in the 1990s when CAF was also used routinely. We now report on studies to determine whether VMD or CAF is the more effective technique for the detection of marks on fabrics. Four different fabrics, nylon, polyester, polycotton and cotton, were utilised during this study, along with 15 donors who ranged in their age and ability to leave fingermarks, from good to medium to poor, thus reflecting the general population. Once samples were collected they were kept for a determined time $(1,2,3,4,5$, $6,7,14,21$ or 28 days) and then treated using either the gold and zinc metal VMD process or standard cyanoacrylate fuming.

The smoother fabrics, such as nylon, consistently produced greater ridge detail whereas duller fabrics, like cotton tended only to show empty prints and impressions of where the fabric had been touched, rather than any ridge details. The majority of fabrics did however allow the development of touch marks that could be targeted for DNA taping which potentially could lead to a DNA profile. Of the two techniques VMD was around 5 times more effective than $\mathrm{CAF}$, producing a greater amount of ridge detail, palmar flexion creases and target areas on more samples and fabrics.
\end{abstract}

Keywords: vacuum metal deposition; cyanoacrylate fuming; fingermarks; fabrics; ridge detail; palmar flexion creases

\section{Introduction}

Fabric is a notoriously difficult substrate from which to acquire and visualise latent prints and, even though several techniques have been investigated, the UK Home Office Manual indicates that there is "no proven process" of developing latent fingermarks on fabrics [1]. Recently, we reported on the visualisation of fingermarks and grab impressions on fabrics using gold/zinc vacuum metal deposition (VMD) [2] and the use of silver VMD in a similar process but on dark fabrics [3]. The current study concentrates on a comparison of the two techniques, vacuum metal deposition (VMD) and cyanoacrylate fuming (CAF), in order to determine which methodology is the most effective for the visualisation of planted fingermarks and grab impressions on selected fabrics. 
VMD, the older of the two techniques, was first reported in the 1960s [4] and its first use in an operational sense was described for polythene by Kent in 1976 [5]. Later, CAF was established by several groups in Japan, Britain, and Canada as a method for developing latent fingermarks [6,7]. While both VMD [8] and CAF [9] are effective methods of visualising latent prints on non-porous surfaces such as plastics and glass, VMD has the advantage that it can develop fingermarks on articles that have been wet or aged. However, VMD does have high start-up costs, needs an experienced operator and can only process one sample at a time. In comparison, CAF tends not to work well on old prints or those subjected to "harsh environmental conditions" [10] but CAF is cheaper to set up and run, multiple samples can be processed at one time, the system can be automated [1] and, in some cases, may be used at crime scenes [11]. These benefits explain why, generally, CAF is more widely used in the development of latent prints compared to VMD.

With VMD (deposited metal) and CAF (polymer), the visualant adheres to, or interacts, with different components of the fingerprint residues, so the appearance of the visualised latent fingermark, even on the same substrate, appears quite differently. VMD will usually visualise negative prints with the valleys adopting the colour of the vaporised metals applied, such as grey when gold then zinc are used, and the ridges, as they are protected by the fingerprint residues, will be the colour of the background substrate $[8,12]$. CAF will visualise positive prints due to the cyanoacrylate polymerisation being catalysed by the water and sodium chloride in the fingerprint deposits forming a white polymer [13]. Since, the polymer does not tend to form on the background substrate the fingermarks stand out from the surface as a visible print. One disadvantage of CAF is that if the surface is of a light colour the contrast may not be enough and therefore further enhancement with fluorescent dyes, such as basic yellow 40 , is necessary [6].

Direct comparisons of both techniques have been carried out previously. VMD was found to produce about $12 \%$ more prints than CAF treated with basic yellow 40 on polyethylene bags [14]. However, more recent work shows that CAF is in fact more effective than VMD on modern plastic packaging, due to changes in the CA composition since the 1980s [15]. VMD was around $17 \%$ more effective than the use of CAF followed by fluorescent dye fuming for low density polyethylene [16] and the use of VMD alone, CAF alone and CAF and VMD in sequence was reported for glass slides [17]. While both VMD and CAF are more traditionally used on smooth surfaces such as plastics, several studies dating back to the 1970s [4] showed that VMD had different levels of efficacy on fabrics. The problematical nature of fabrics was also reported [18] and the limited success obtained depended on the fabrics having a smooth clean fine weave surface, such as seen with silk or nylon. 
To our knowledge no work has been reported on the use of CAF with fabrics, therefore this study was designed to determine whether VMD or CAF was the more successful in the visualisation of latent fingermarks on these surfaces.

\section{Materials and Methods}

The fabric types used in this study were cotton, nylon, polyester and polycotton (60 $\%$ cotton and $40 \%$ polyester mix) and all were white in colour. All the fabrics complied with the Home Office requirement of a minimum of 3 threads per $\mathrm{mm}$ on fabrics being examined for fingermark development [1]. The fabrics were prepared for deposit collection by cutting $23 \mathrm{~cm} \times 16 \mathrm{~cm}$ sized samples that were labelled with the fabric type, hand position ( $\mathrm{F}$ fingers, $\mathrm{P}-$ palm), donor number, and process day. These specimens were then kept in plastic wallets until they were processed with either gold/zinc VMD or CAF/BY40.

The 15 donors used in this study were a mix of males and females who ranged in age (35 to 60) and their potential to leave fingermark deposits (previously graded on paper). Prior to collection, the donors had not washed their hands for at least 45 minutes so the deposits left were "normal" and not "loaded". The fabric swatch was laid on the collector's arm and the donor "grabbed" the sample firmly for 10 seconds thus depositing a mark. Only one sample was collected at a time, with a minimum of 45 minutes between collections if more than one sample was collected in a day, thus allowing for replenishment of fingerprint residues. After acquisition, the samples were placed in plastic wallets, in the dark, at room temperature for 1 to $7,14,21$ or 28 days and were then processed. Overall, 600 samples, consisting of 15 donors $\times 10$ days $\times 4$ fabrics $\times 2$ techniques (VMD and CAF) were acquired.

The VMD equipment used in this study was a round chambered Edwards 24" Metal Deposition Unit, and was operated as described previously [2]. The CAF samples were processed in a Mason-Vactron unit (MVC3000) using standard operating procedures. The fabric samples were hung in the cyanoacrylate fuming cabinet and ethyl cyanoacrylate ( $2 \mathrm{~g}$; CSI Ltd. Superglue) was added to the aluminium foil dish in the heater. The fuming was commenced using a standard 45 -minute cycle with a humidity of $80 \%$ and temperature of $120{ }^{\circ} \mathrm{C}$ ( 15 min relative humidity cycle, $15 \mathrm{~min}$ superglue cycle and $15 \mathrm{~min}$ purge cycle). The samples were processed by dipping in Basic Yellow 40 solution ( $2 \mathrm{~g}$ in $1.0 \mathrm{~L}$ of ethanol) for 1 $\mathrm{min}$, then rinsed under running tap water until the water ran clear. The fluorescent dye binds to the white CAF polymer and allows the impressions to be visualised under Quaser light (350-469 nm) and they were then photographed using a digital camera fitted with a $476 \mathrm{~nm}$ viewing filter.

The visualised marks were graded, from "No development" to "Excellent", depending on the amount of ridge detail observed:

(0) No development - no visible or recognisable marks on fabric. 
(1) "Empty" prints - where the donor had touched the fabric could be seen but no ridge detail observed on fingertips or palm.

(2) Fair - pattern and ridge flow and/or palmar flexion creases visible, but not enough detail for identification.

(3) Good - ridge characteristics (Galton details) visible on some fingermarks.

(4) Excellent - good ridge detail on all fingertips and palm with visible pores, ridge edge detail and ridge flow.

\subsection{Results and Discussion}

\subsection{Overall fingermark grading on all fabrics}

Considering the overall results of samples on all the fabric types visualised with VMD [Figure 1], it can be seen that cotton is not a fabric that allows VMD development of fingermark ridge detail, as virtually all of the samples are classed as no development or empty apart from 2 at fair (1.3\%) and 1 at good (0.7 \%). The same is true of the cotton samples, visualised with CAF [Figure 2] where $100 \%$ of the cotton samples showed no development. Nylon samples, developed with VMD, range from no development to excellent and it was the fabric with the least number of grade 0 samples (5.3\%) compared to cotton $(48 \%)$, polyester $(25.3 \%)$ and polycotton (29.3\%) [Figure 1]. Nylon was also the only fabric with any grades higher than 0 using CAF (49.3\% at grade 1 and $7.3 \%$ at grade 2) [Figure 2], though this may be in part due to excess background staining caused by the BY40 dye. The polyester VMD samples ranged from grade $0(25.3 \%)$ to one sample $(0.7 \%)$ graded as 4. The CAF samples did not show any ridge detail and all 150 of the samples were graded as 0 . For polycotton with VMD there was a full range of grades: $29.4 \%$ grade 0 , $42.7 \%$ grade $1,13.3 \%$ grade $2,10 \%$ grade 3 and $4.7 \%$ grade 4 but once more all the CAF samples were graded as 0 . 


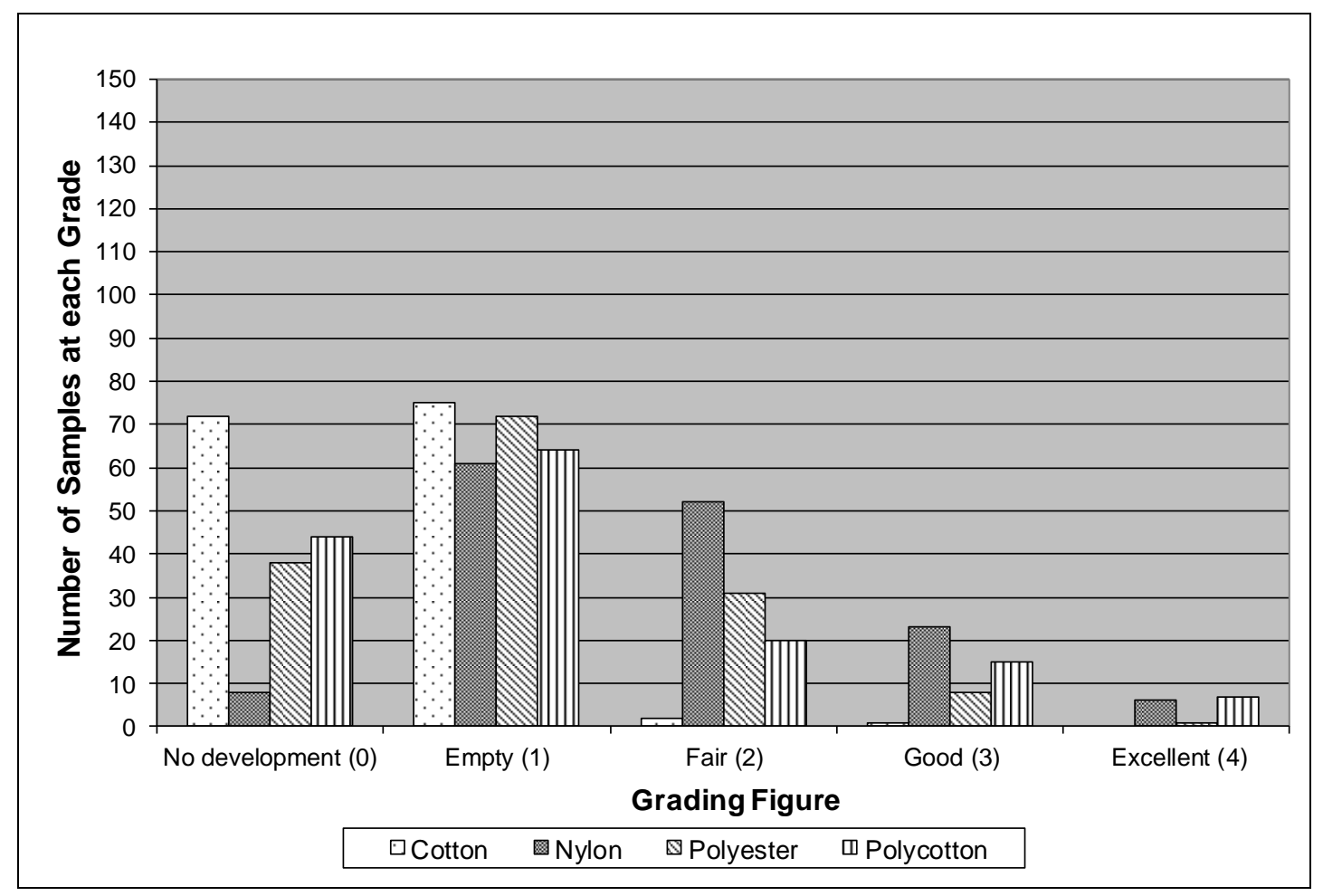

Figure 1 - Overall grading from 0 (No Development) to 4 (Excellent) of samples on all fabric types visualised with VMD.

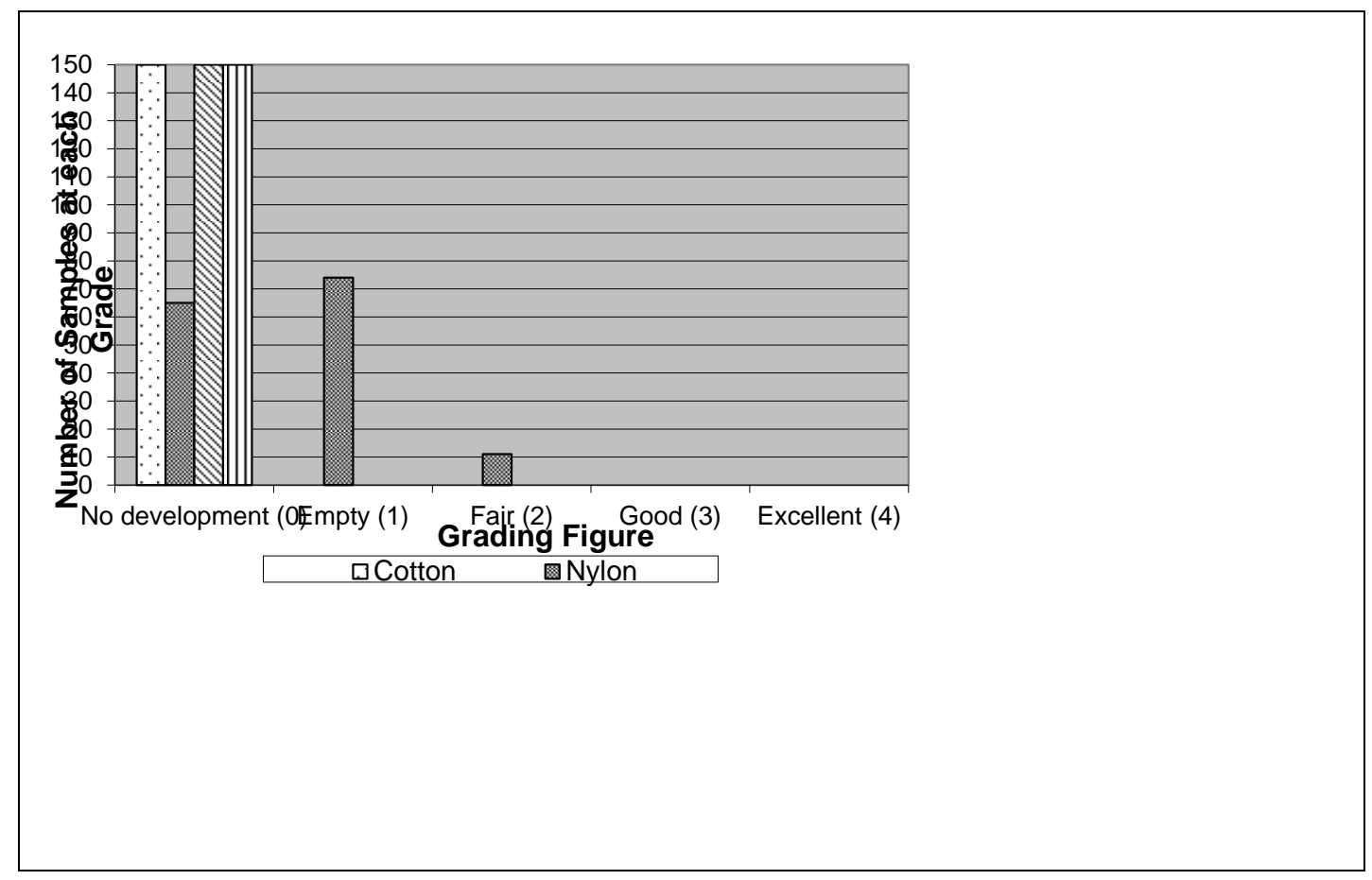

Figure 2 - Overall grading from 0 (No Development) to 4 (Excellent) of samples on all fabric types visualised with CAF.

\subsection{Ridge Detail}


Figure 3 shows that with VMD, cotton only showed one sample with ridge detail (day 1), whereas nylon had 47 samples displaying ridge detail on all of the days except 28. Polyester (15 samples) showed ridge detail on all of the days except days 6 and 28 whilst polycotton (28 samples) allowed visualisation of ridge detail on every day. The nylon VMD samples showed the most detail on days 1 and 2 (9 samples on each day) with the least detail being seen on day 28 (none) and with little detail on days 14 and 21 . This shows that the fresher samples (days 1 and 2) allowed more detail to develop and that detail declines as the samples age. The level of ridge detail left being due to the freshness of the samples is reinforced by considering day 3 where seven nylon, five polyester and six polycotton samples all exhibited some form of ridge detail. Also, the number of ridge detail reduces over time, where on day 21 there are only two samples on nylon and polycotton, and one on polyester that left ridge detail while on day 28 only two polycotton marks containing ridge detail were seen. All this reinforces the view that the order of fabrics for fingermark visualisation is nylon, polycotton, polyester, and cotton when developed with VMD. Figure 4 is a photograph of a day 3 nylon sample visualised with VMD clearly showing ridge detail and palmar flexion creases and Figure 5 is a day 1 sample on cotton visualised with VMD, displaying empty prints, though some palmar flexion creases can be observed.

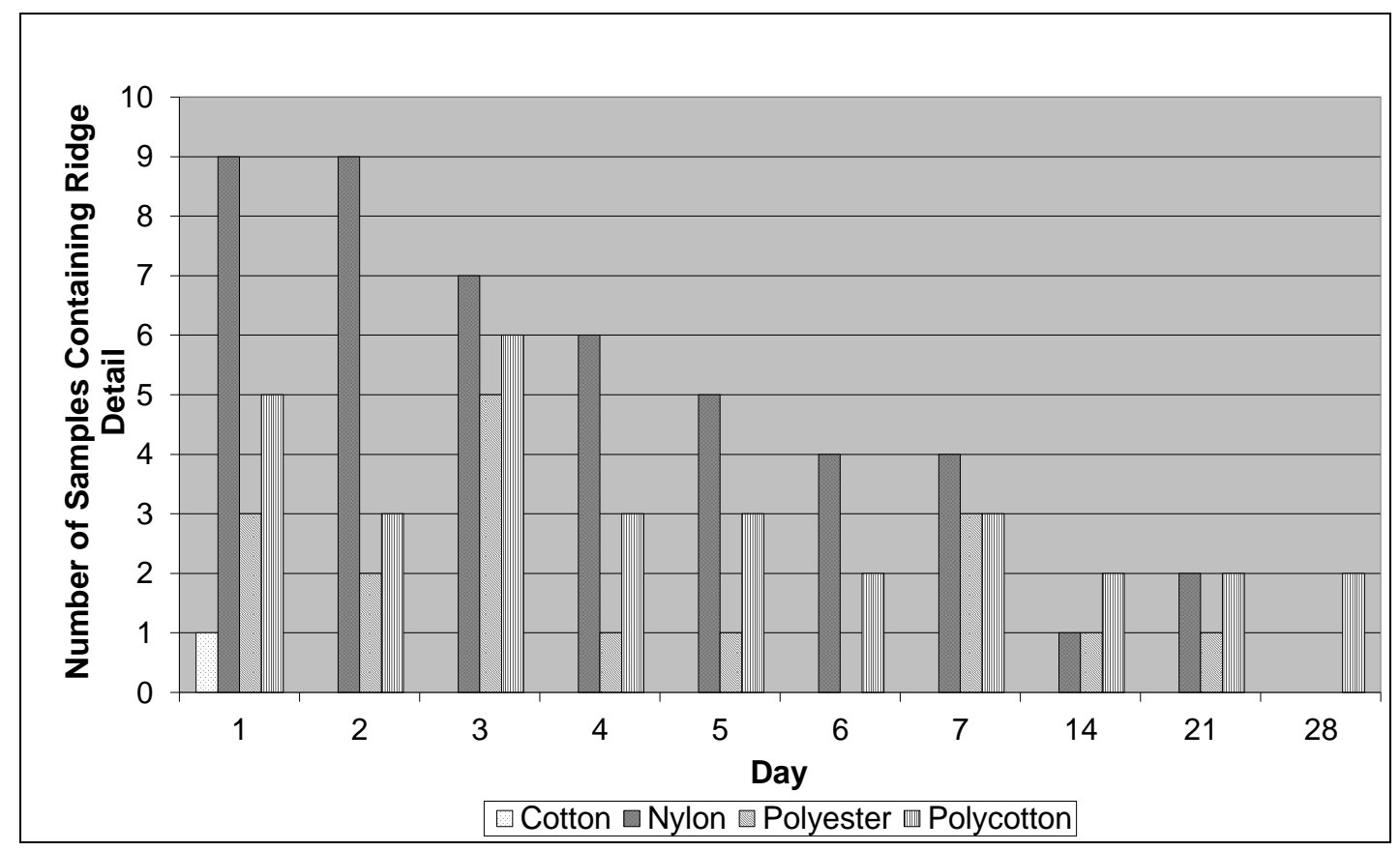

Figure 3 - Number of cotton, nylon, polyester and polycotton samples containing ridge detail using VMD.

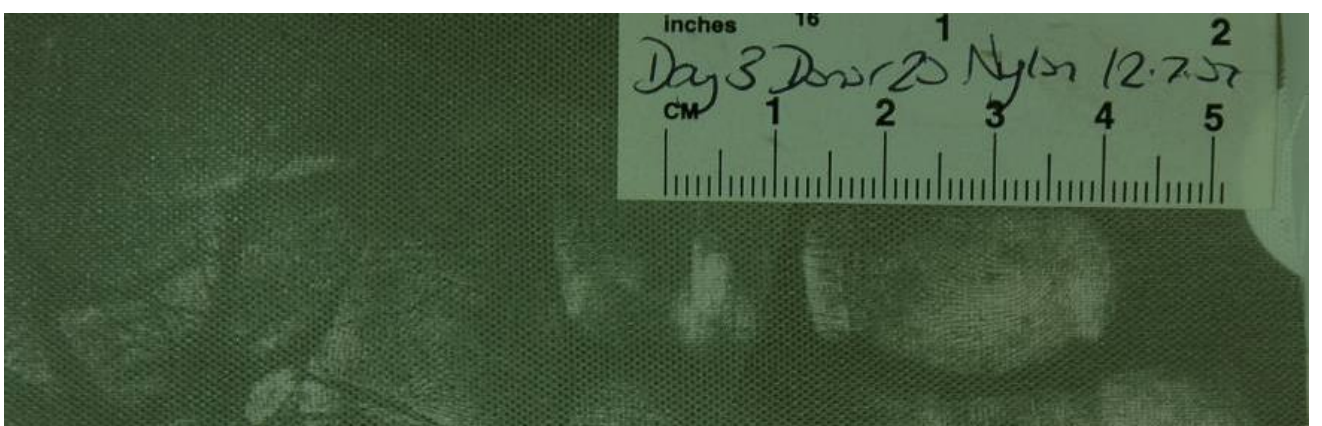


Figure 4 - Day 3 sample on nylon visualised with VMD containing ridge detail and palmar flexion creases.

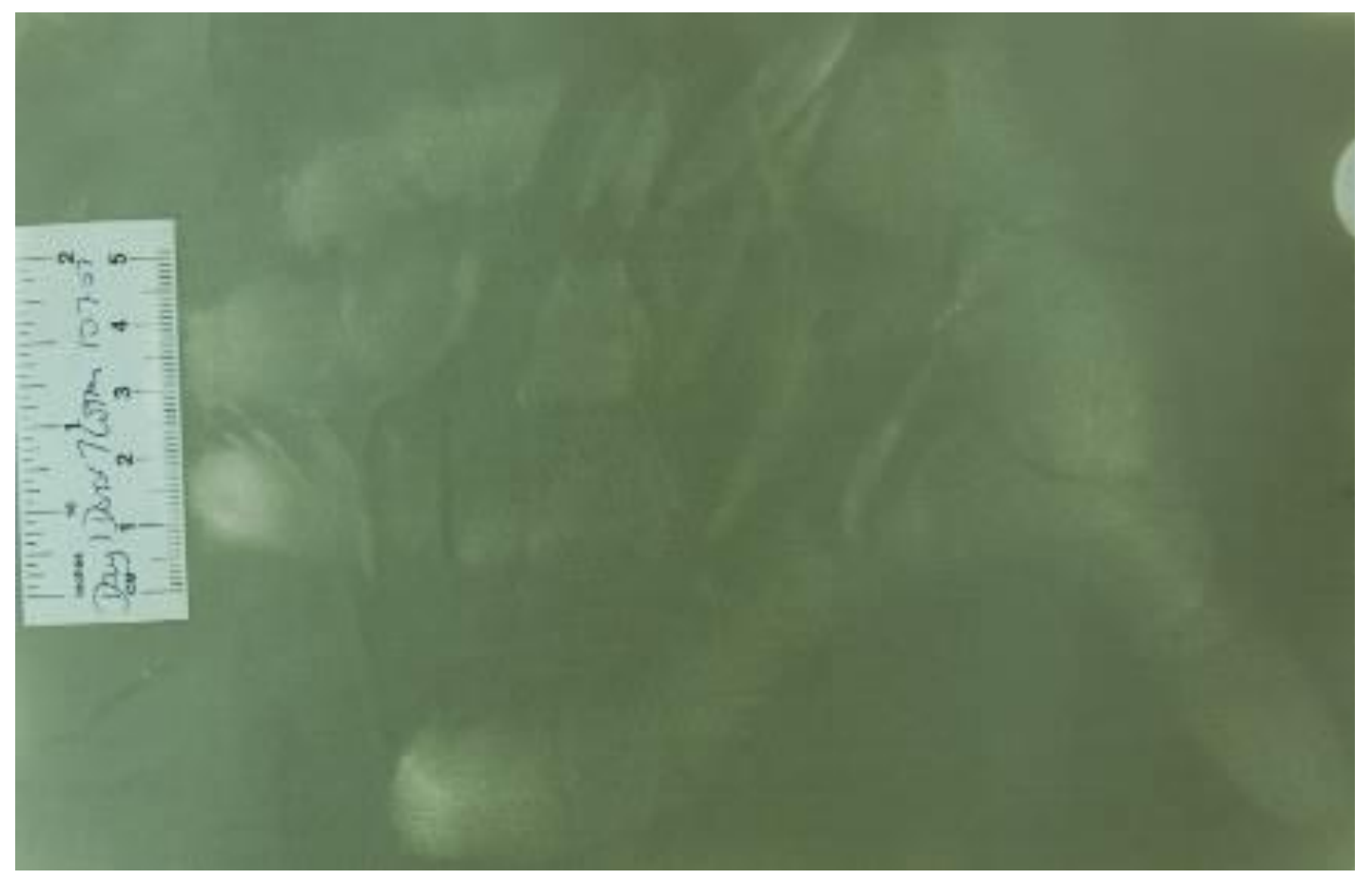

Figure 5 - Day 1 sample on cotton visualised with VMD displaying empty prints, with some palmar flexion creases.

With CAF there were few marks observed and the majority of cases (515 or $85.8 \%$ ) were graded as no development at all [Figure 6] though 54 (9\%) of those samples had 
possible deposits and thus target areas. Of the nylon samples $74(12.3 \%)$ showed a target area with no ridge detail and the remaining samples $(11 ; 1.8 \%)$ had some detail (5 samples on day two, 3 on day 6 and only one on days 1, 7 and 14). Some of this could be explained by results of the Archer et al. study [19] where it was concluded that there was an increase in fatty acids in the prints for the first ten days followed by a decline, then another increase later. A similar variation is seen here where there is a period (days 3-5) where no marks were visualised but then some could be seen on days 6,7 and 14 but none thereafter.

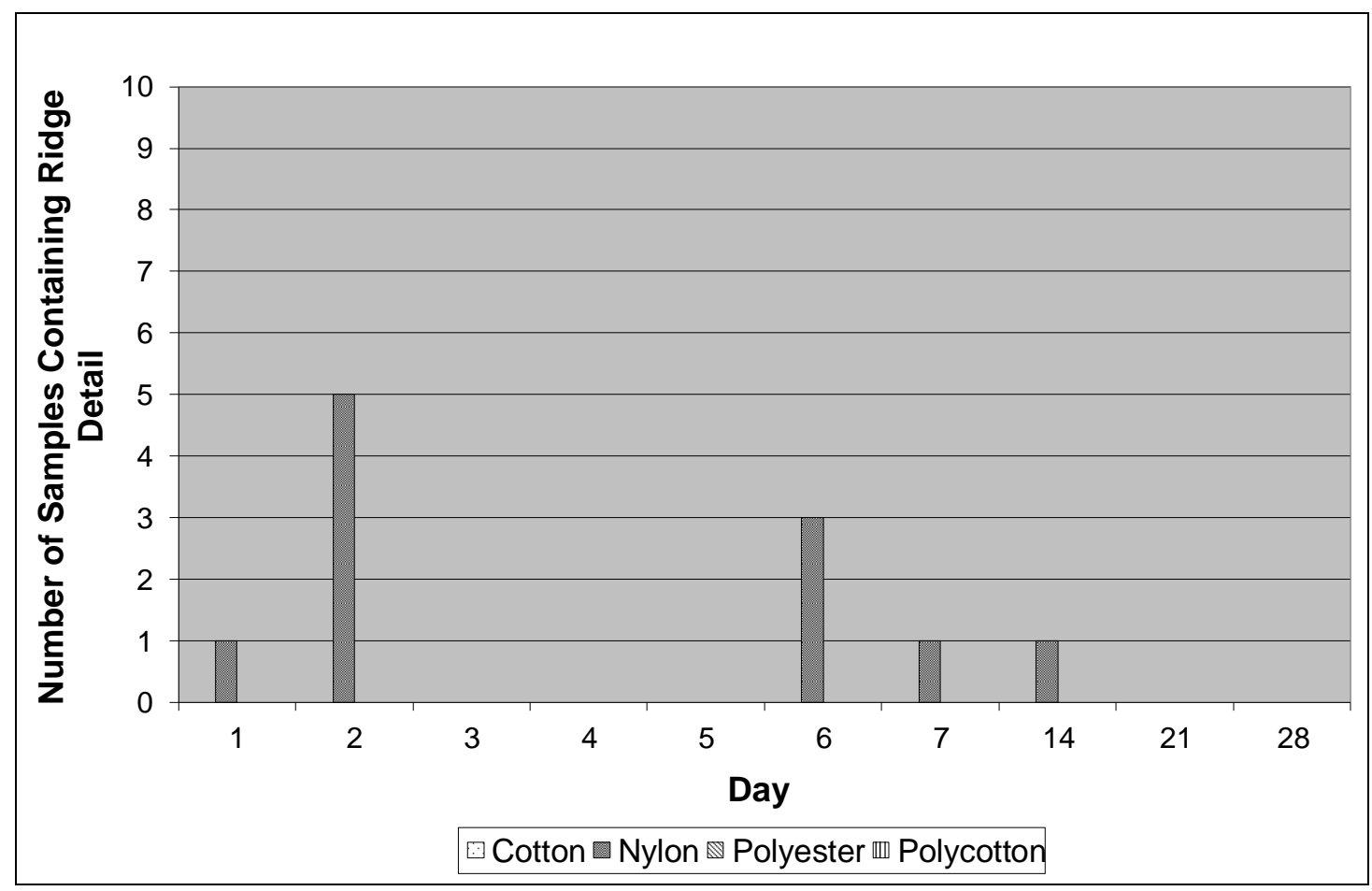

Figure 6 - Number of cotton, nylon, polyester and polycotton samples containing ridge detail using CAF.

Overall, the differences between the fabrics and the amount of ridge detail visualised could either be due to the fabric allowing the fingerprint residues to evaporate more readily or that the fabric is absorbent and therefore the residues deposited from the donors' hands not remaining on the fabric surface. This could result in there being no visualisation due to absorption of the fingerprint residues in both the VMD and CAF processes. When comparing all the fabrics it appears that the smoother the fabric and those with possible surface coatings more readily ridge detail is observed.

\subsection{Samples containing palmar flexion creases}

Figure 7 demonstrates that when using VMD all the fabric samples had palmar flexion creases on all 10 days. The amount of cotton samples with palmar flexion creases varied from only one in a day (days 2, 3, 5, 7, 14 and 21) to three in a day (1 and 28). The amount 
of nylon samples with palmar flexion creases ranged from only three in a day (28) to eight in a day (3). With polyester there was a high of 7 out of 15 on day 7 to lows of 2 (days 3,4 and 21) and 1 on day 28, whereas polycotton had six marks on days 1 and 7, followed by five on days 2, 3 and 5, decreasing to two on days 4 and 14 . There were no days where all the samples were positive for palmar flexion creases.

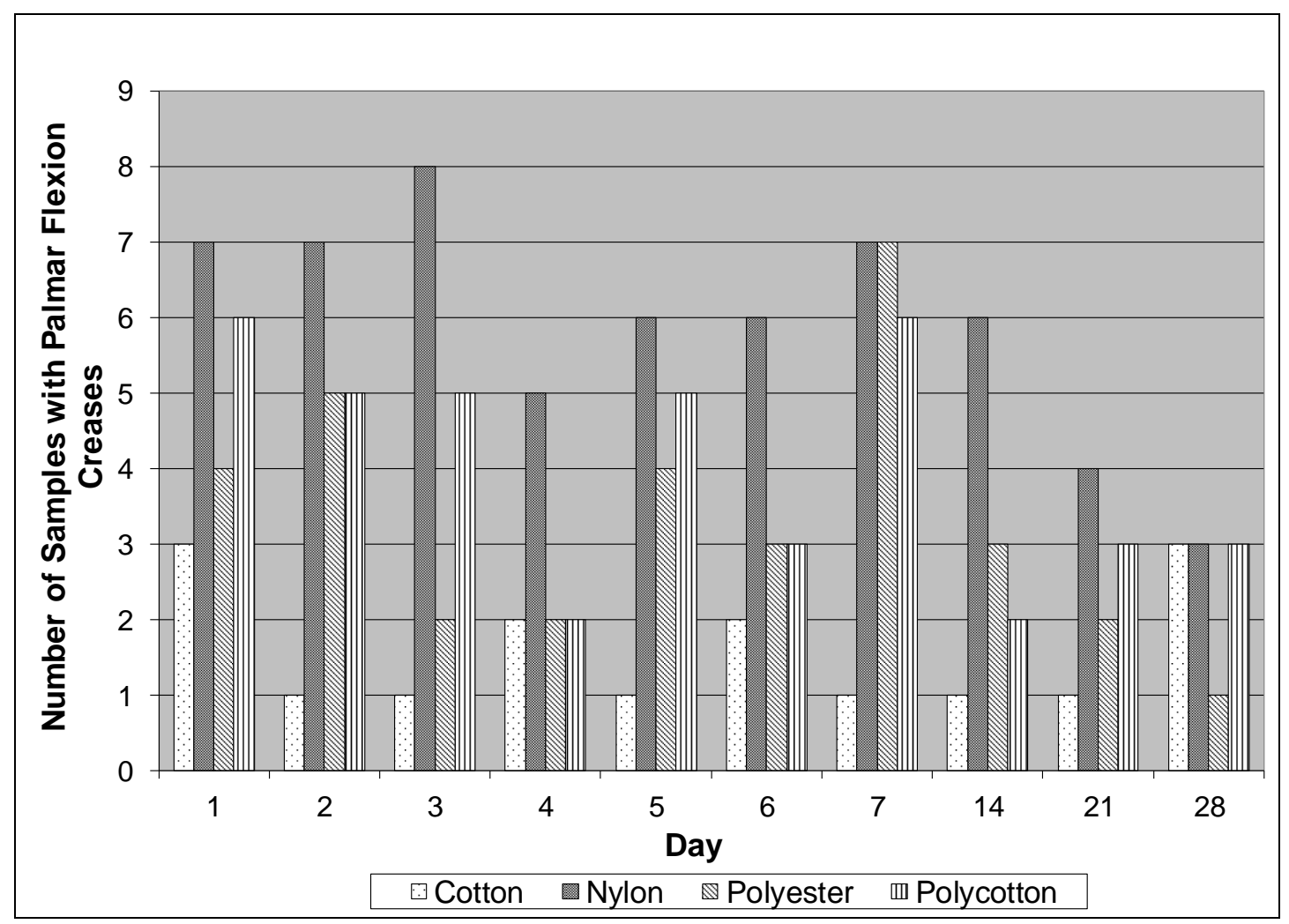

Figure 7 - Number of cotton, nylon, polyester and polycotton samples containing palmar flexion creases on VMD samples.

However, with CAF [Figure 8] only nylon showed palmar flexion creases. The results ranged from seven samples on day 7, six on days 2 and 6, five on day 21, three on days 5 , 14 and 28 and only one sample on days 1, 3 and 4 . Therefore, this reinforces the idea that it is the smoother surface on nylon fabric that allows the residues to remain on the surface rather than penetrating the fabric or evaporating, which in turn allows the CAF to develop grab and fingertip impressions. 


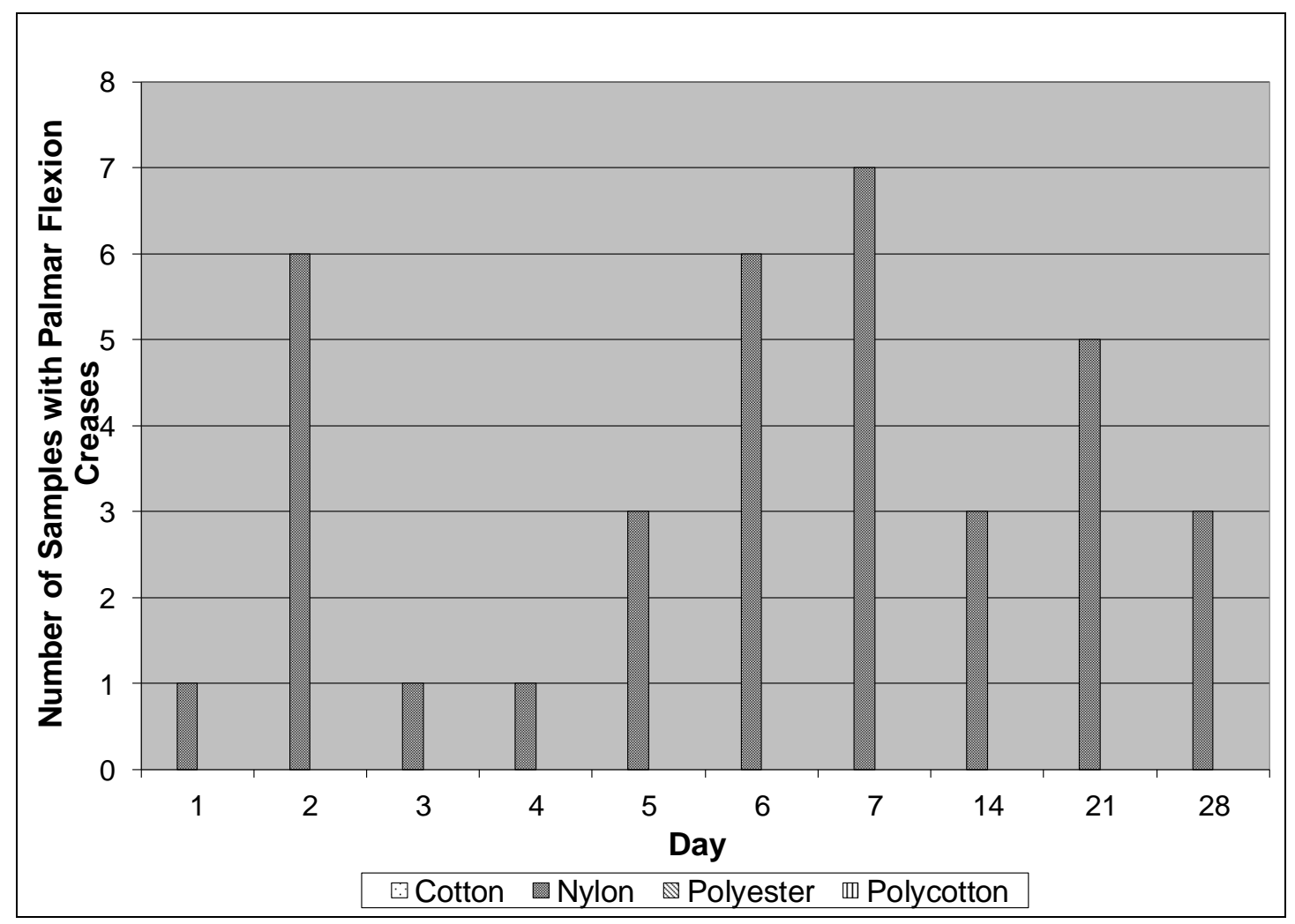

Figure 8 - Number of cotton, nylon, polyester and polycotton samples containing palmar flexion creases on CAF samples.

\subsection{Donor grading}

Interestingly, the donors can be graded into five categories related to their ability to leave marks on the different fabric types. The majority of donors were classed as poor on all the fabrics except nylon which had half the samples graded as poor and the other half split between the other four categories. With cotton fourteen out of fifteen donors were classed as poor, with only one donor rated as medium, though this is probably a spurious result due to ridge detail being observed in this instance. Polyester also had the majority of donors graded as poor (eleven), with the remaining five samples divided amongst medium to poor and good to medium. With polycotton ten donors were classed as poor, with the other five donors spread over the remaining categories. Finally, nylon had the least number of poor donors and the most even spread across all five categories. 


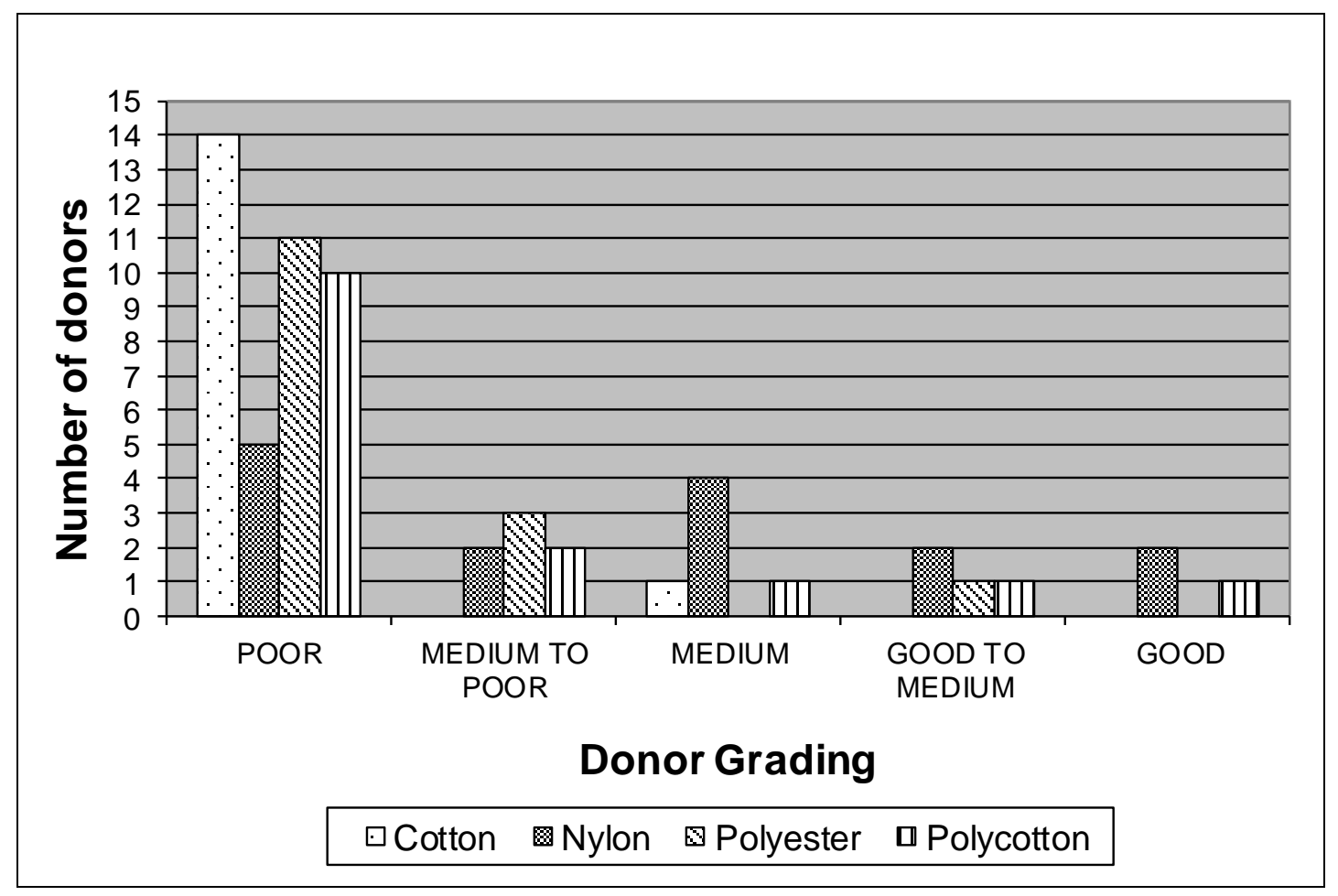

Figure 9 - Donor grading for all donors on cotton, nylon, polyester and polycotton using VMD.

With CAF it was only nylon that had any development of ridge detail though it did not produce many significant results with only ten samples showing ratings of two. Therefore, all the donors for the other four fabrics were graded as poor, while nylon had a mixture of the first three categories. The samples did not produce any fingermark ridge detail, but did have some palmar flexion creases, which could be targeted for DNA, therefore even though the marks themselves cannot be used to identify an assailant, if a DNA profile is produced, this may lead to identification. 


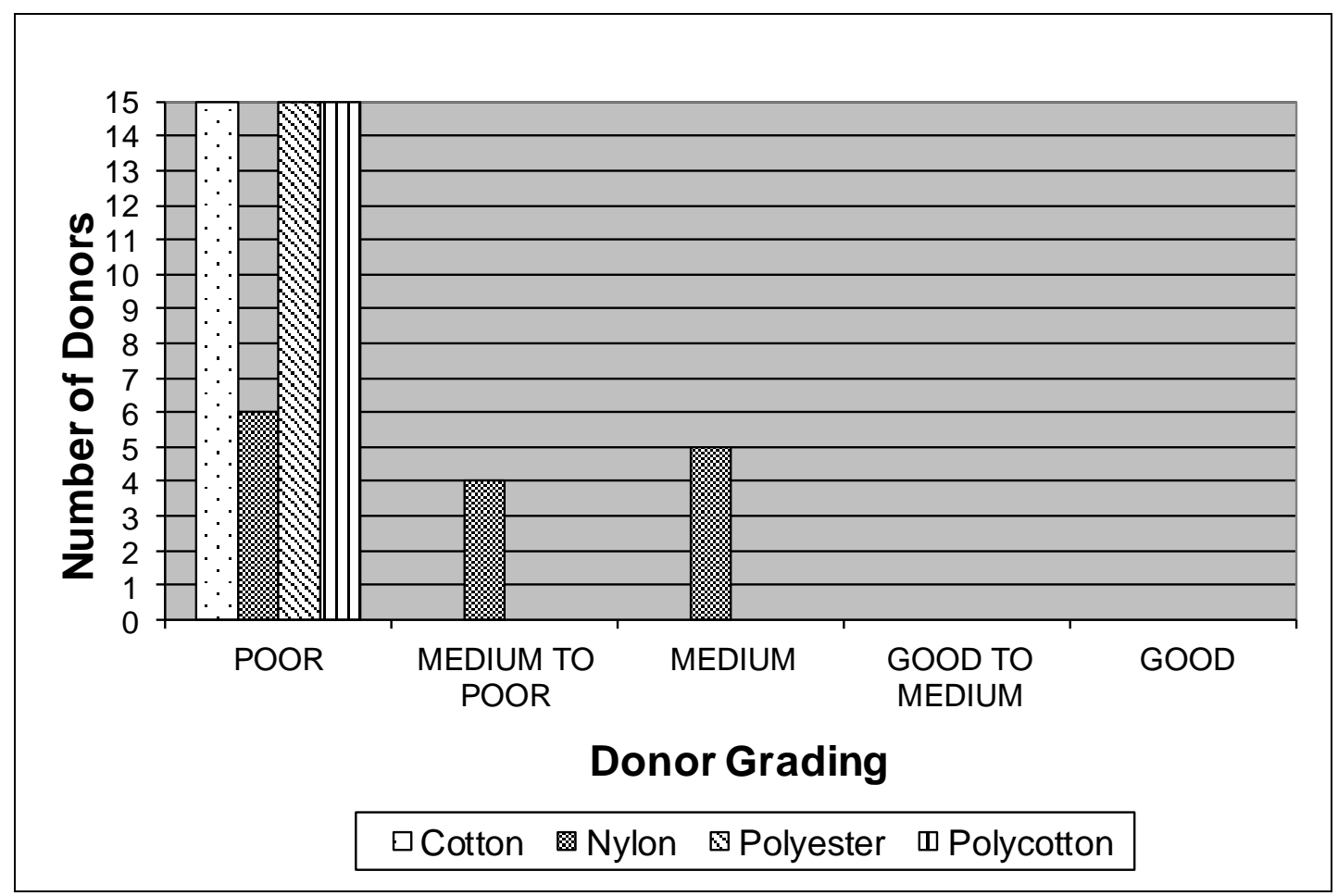

Figure 10 - Donor grading for all donors on cotton, nylon, polyester and polycotton using CAF.

Overall, these results show that it is a combination of the donor's ability to deposit fingerprint residues and the type of fabric on how much detail is visualised. The donors themselves ranged in age and were a mixture of males and females, however even though it may be expected that as donors age the level of fingerprint residues produced would decline, it was not always evident in this study, as some older donors laid marks that visualised greater detail than some of the younger donors [20]. When looking at the samples analysed and their donor ratings, nylon is the smoothest fabric and only 5 out of 15 donors were classed as poor with VMD and 6 out of 15 graded as poor for CAF. The only conclusions that can be drawn about the amount of detail left on each fabric is that with nylon, information can be obtained using both VMD and CAF but in the case of polycotton, only VMD seems to be effective in finding ridge detail. However, if a cotton or polyester fabric had been tested the day after the incident and the assailant was an extremely good donor some detail might be found.

\subsection{Target areas over time}

It can clearly be seen from Figure 11, that every fabric visualised with VMD has target areas on every day, but it is, once more, nylon that provides the largest number with six of the days $(2,3,4,6,7$ and 21). With nylon the lowest number of samples to have target areas was on day 28 where $80 \%$ left marks and this is only $6.7 \%$ lower than the highest value of $86.7 \%$ for cotton. Most samples to have target areas on cotton was on day 
1; this number fell to $60 \%$ by day 2 and decreased from a maximum of $66.7 \%$ to a minimum of $40 \%$ indicating that nylon allows more information to be visualised than cotton. Neither polyester nor polycotton have a $100 \%$ day though both have days where fourteen (93.3\%) samples produced a target area - polyester on day 3 and polycotton on days 2 and

3. The rest of the days ranged from a low of $60 \%$ (polyester - days 4 and 21 and polycotton - day 28 ) to a high of $86.7 \%$ (polyester - day 2 and polycotton - day 1 ).

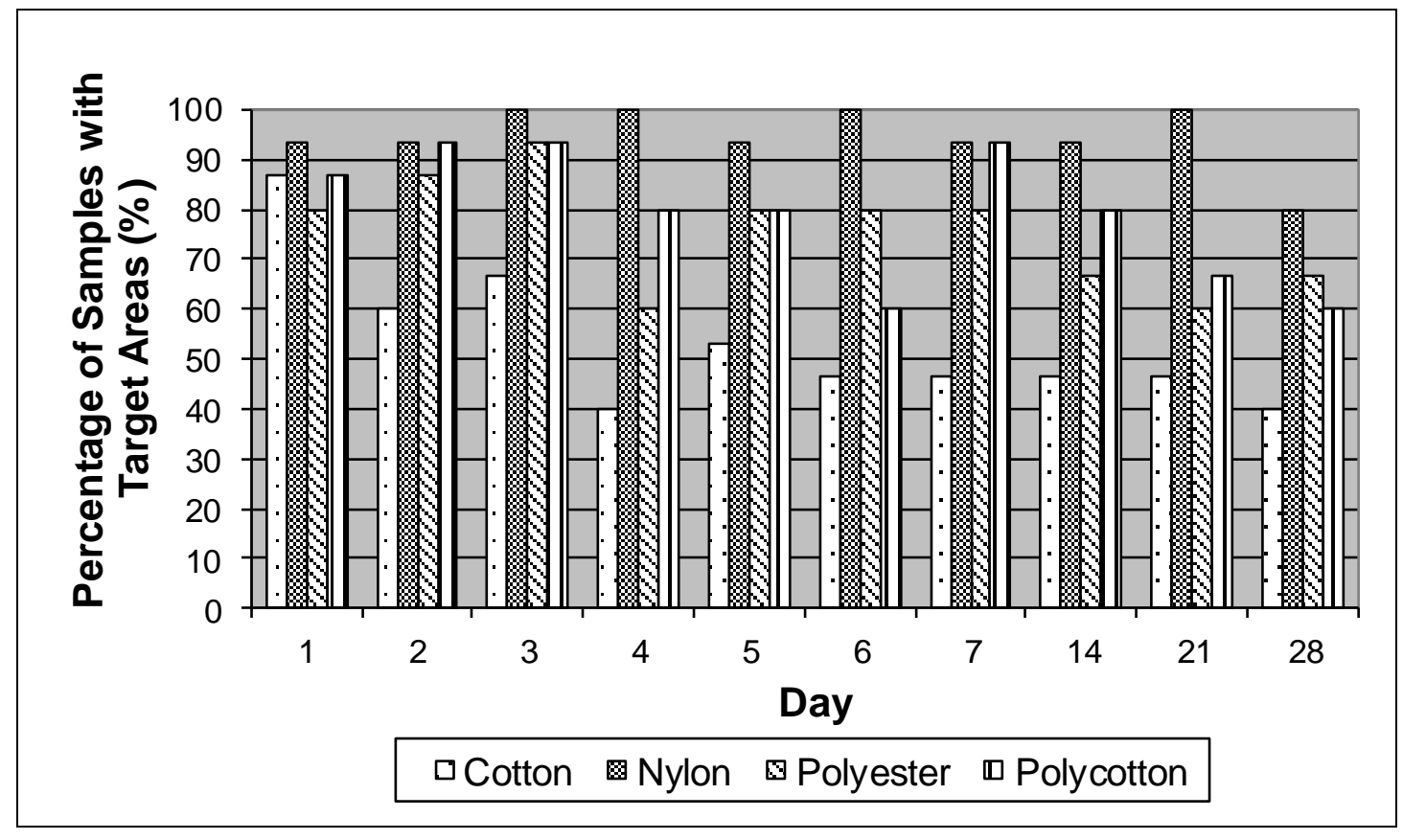

Figure 11 - Percentage of samples with target areas on cotton, nylon, polyester and polycotton samples with VMD

With CAF [Figure 12] nylon was the only fabric that had target areas on every day ranging from $100 \%$ of the samples on day 14 to only $53.3 \%$ on day 5 . Of the 150 nylon samples there were only 32 that showed no impressions and these were spread across all the days from day 1 to day 28 . This demonstrates that target areas can be developed on nylon over long periods from day one to twenty-eight days after an incident. Though days 1 and 2 had $93.3 \%$ of samples with target areas this dropped to $53.3 \%$ on day three. However, after day 6 the samples with target areas again increased up to $80 \%$ and above, only falling to $66.7 \%$ on day 28 . Therefore, this indicates that impressions and target areas may be visualised over long periods.

Of the remaining $450 \mathrm{CAF}$ samples (cotton, polyester, and polycotton) only 21 developed target areas. Cotton only had two samples on day 3, polycotton had one sample on day 1 and one on day 21 , whereas polyester had the most target areas, with 17 . 


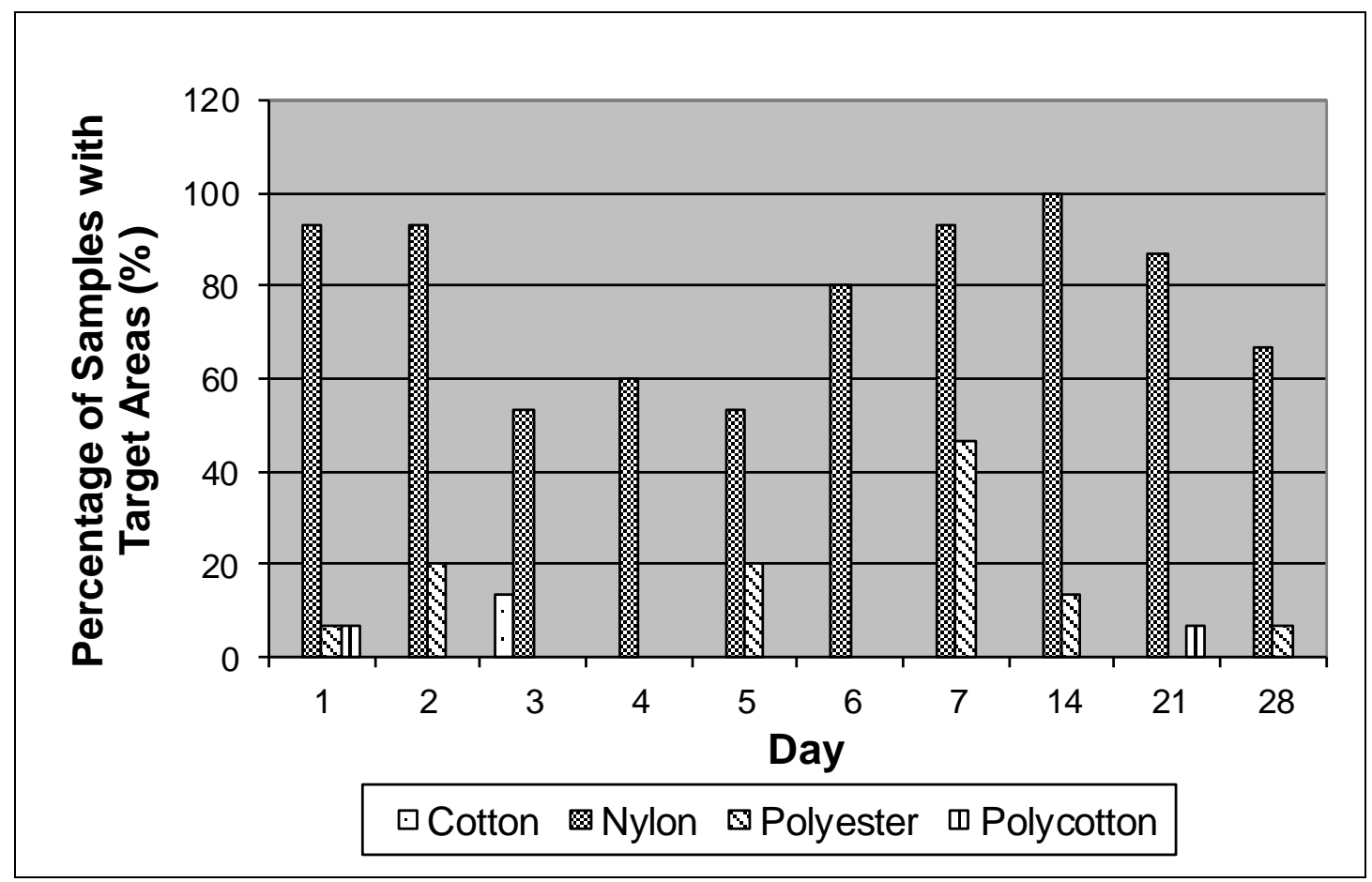

Figure 12 - Percentage of samples with target areas on cotton, nylon, polyester and polycotton samples with CAF

\section{Conclusion}

Nylon demonstrates a better ability to retain fingerprint residues and therefore allows more ridge detail to be developed when VMD is used. However, all the fabrics have the ability to develop impressions, from small fingertip marks to full grabs that could be utilised as target areas for DNA. With CAF there was a lack of impression development on all the fabrics, which illustrates that the duller fabrics do not allow the fingerprint residues to remain on the fabric surface long enough for the CAF to adhere to them and allow visualisation. Therefore based on these results, it would seem that smoother manmade fabrics are better at allowing visualisation of impressions by means of CAF. To confirm this there will need to be further research carried out utilising more manmade and natural fabrics and work is ongoing in this area.

Of the two techniques, VMD produced more ridge and palmar flexion detail than CAF. Other useful information, such as partial ridge detail and palmar flexion creases was also obtainable, with VMD giving $73 \%$ of some form of visualisation whilst CAF was only $14 \%$. Overall, both techniques produced some marks that could be used to identify, or at the least include or exclude, an individual from an investigation, with VMD being five times more likely to provide such information compared to CAF. 


\section{Acknowledgements}

The authors would like to thank all the donors who donated time and prints to this study, along with the SPA staff at the Dundee for their time and expertise.

\section{References}

[1] V. Bowman (ed.) Manual of Fingerprint Development Techniques, $2^{\text {nd }}$ ed., White Crescent Press Ltd Luton, 1998 ( $3^{\text {rd }}$ revision, December 2009).

[2] J. Fraser, K. Sturrock, P. Deacon, S. Bleay, D.H. Bremner, Visualisation of fingermarks and grab impressions on fabrics. Part 1: Gold/zinc vacuum metal deposition, For. Sci. Int. 208 (2011) 74-78.

[3] S. Knighting, J. Fraser, K. Sturrock, P. Deacon, S. Bleay, D.H. Bremner, Visualisation of fingermarks and grab impressions on dark fabrics using silver metal deposition, Sci. Just. 53 (2013) 309-314.

[4] D. S. Hambley, The physics of vacuum evaporation development of latent fingerprints, PhD Thesis, Department of Physics, Royal Holloway College, March 1972T.

[5] T. Kent, G.L. Thomas, T.E. Reynoldson, H.W. East, A vacuum coating technique for the development of latent fingerprints on polythene, J. For. Sci. Soc. 16 (1976) 93-101.

[6] Bandey, $H$. and Kent, T. Superglue treatment of crime scenes a trial of the effectiveness of the Mason Vactron superfume process, Home Office, St. Albans, 2003.

[7] C. Champod, C. Lennard, P. Margot, M. Stoilovic, Fingerprint and other ridge skin impressions, CRC Press, Boca Raton, 2004.

[8] N. Jones, M. Stoilovic, C. Lennard, C. Roux, Vacuum metal deposition: developing latent fingerprints on polyethylene substrates after the deposition of excess gold, For. Sci. Int. 123 (2001) 5-12.

[9] L.A. Lewis, R.W. Smithwick III, G.L. Devault, B. Bolinger, S.A. Lewis, Processes involved in the development of latent fingerprints using the cyanoacrylate fuming method, J For. Sci. 46(2001) 241-246.

[10] S.P. Wargacki, L.A. Lewis, M.D. Dadmun, Understanding the chemistry of latent fingerprints by superglue fuming, J. For. Sci. 52 (2007) 1057-1062.

[11] P. Tissier, J.C. Didierjean, C. Prud'Homme, J. Pichard, F. Crispino,.A cyanoacrylate case for developing fingerprints in cars, Sci. Just. 39 (1999) 163-166.

[12] D. Philipson, S. Bleay, Alternative Metal Processes for Vacuum Metal Deposition, J. For. Ident. 57 (2007) 252-273.

[13] S.P. Wargacki, L.A. Lewis, M.D. Dadmun, Enhancing the quality of aged latent fingerprints developed by superglue fuming: loss and replenishment of initiator, J. For. Sci. 53 (2008) 1138-1144.

[14] T. Kent, Recent research on superglue, vacuum metal deposition and fluorescence examination, Home Office, PSDB, 1990. 
[15] R. P. Downham, S. Mehmet, V. G. Sears, A pseudo operational investigation into the development of latent fingerprints on flexible plastic packaging films, J. For. Ident. 62 (2012) 661-682.

[16] A.H. Misner, Latent fingerprint detection on low density polyethylene comparing vacuum metal deposition to cyanoacrylate fume and fluorescence, J. For. Ident. 42 (1992) 26-33.

[17] N.E. Masters, J.D. DeHaan, Vacuum metal deposition and cyanoacrylate detection of older latent prints, J. For. Ident. (1996) 32-46.

[18] A.H. Misner, Fingerprint detection using vacuum metal deposition, Canadian Police Research Centre. 1993

[19] N.E. Archer, Y. Charles, J.A. Elliott, S. Jickells, Changes in the lipid composition of latent fingerprint residue with time after deposition on a surface, For. Sci. Int. 154 (2005) 224-239.

[20] Yamashita, B. and French, M. 2011. Latent print development. In: McRoberts, A. ed. The fingerprint source book. Washington: U.S. Department of Justice. 2011, pp. 7-1 7-67. 